\title{
Standard Model Higgs in the Inflationary Universe
}

\author{
Kohei Kamada*t \\ School of Earth and Space Exploration, Arizona State University \\ E-mail: kohei.kamadalasu.edu
}

\begin{abstract}
Recent results of collider physics suggests that the Standard Model Higgs potential is metastable if there are no physics beyond the Standard Model. If it is really metastable, high scale inflation is problematic since the Standard Model Higgs is destabilized during inflation and falls down to the unwanted true vacuum. Here we consider the nontrivial interaction of the Standard Model Higgs to generate additional mass term for it during inflation. We show that this helps for the our electroweak vacuum to be selected. Moreover, we point out that the additional mass term does not have to be larger than the Hubble parameter during inflation. This article is based on the paper Ref. [1].
\end{abstract}

11th International Workshop Dark Side of the Universe 2015

14-18 December 2015

Yukawa Institute for Theoretical Physics, Kyoto University Japan

* Speaker.

${ }^{\dagger}$ A footnote may follow. 


\section{Introduction}

Recent results at the Large Hadron Collider (LHC) [2,3] can be well explained by the Standard Model (SM) with a $125 \mathrm{GeV}$ Higgs boson and thus far there is no concrete evidence of new physics beyond the SM. On the other hand, according to the current measurements of the Higgs and top quark masses [4], it is likely that the SM Higgs potential becomes negative at $h>10^{10} \mathrm{GeV}$ and the electroweak vacuum is metastable if there is no physics beyond the SM [5]. It may be an important hint for high-energy physics.

Once we assume that the electroweak vacuum is metastable, we might face several problems in cosmology. The current data suggests that the lifetime of the electroweak vacuum is longer than the age of the Universe [6], and there is no constraint on the reheating temperature from the thermalfluctuation-triggered electroweak vacuum decay [5]. However, in the very early Universe, the story can be different. One of the important ingredients in modern cosmology is inflation, which solves many problems in the early Universe such as the flatness and horizon problems. In this case, the minimal explanation of the Universe is to add one scalar field to drive inflation, without strong interaction with the SM particles. During inflation, the vacuum fluctuation in the quasi-de Sitter background of the Higgs field may also push it to the unwanted Anti de Sitter (AdS) vacuum if the Hubble parameter is too large. Thus, it may spoil inflation or, at least, our Universe that lands in the metastable vacuum may be unlikely [5, 7]. Therefore, low-energy scale inflation may be favored in this viewpoint.

This problem can be avoided by supposing a small coupling between inflaton and the SM Higgs field without giving any major effects on the dynamics of inflaton. This is because the coupling produces the "Hubble-induced mass" during inflation, which pushes the field value where the Higgs potential goes negative to a much larger value. If the induced mass is much larger than the Hubble parameter, the Higgs field is stabilized at the origin and its fluctuations is sufficiently suppressed. On the other hand, if the induced mass is smaller than the Hubble parameter, it seems to be difficult to suppress the quantum fluctuations and hence we suffer from the unwanted vacuum decay in the case of high-scale inflation.

In this article, however, we point out that we can construct a scenario with a high-scale inflation in which most part of the Universe can avoid the vacuum decay while the induced mass is not so large if the number of $e$-folds during inflation is not too large. This is because the evolution of the expectation value of the Higgs field during inflation is suppressed and it can be smaller than the field value of the potential barrier if the Hubble-induced mass $m_{H}$ is large enough, $\Delta m_{h}^{2} / H_{\text {inf }}^{2} \gtrsim 2 \times 10^{-2}$ and the number of $e$-folds during inflation is not too large. In addition, if the reheating temperature is high enough, the present Universe can be safely realized. Note that after inflation the Higgs field still slow-rolls and the time-dependent potential barrier may catch it up. The Higgs field will roll down towards the unwanted AdS vacuum in this case. If the Higgs field is thermalized before being caught up by the potential barrier, the Higgs field safely settles down to the electroweak vacuum. Owing to a relatively high reheating temperature, the Higgs field is thermalized earlier. Here we give a rough estimate for such a healthy scenario. We also point out that it would be possible to verify such a high reheating temperature by the future gravitational wave experiments. 


\section{Fluctuation of the Higgs field with a small induced mass during inflation}

At the large field values $h \gg v \equiv 246 \mathrm{GeV}$, the SM Higgs potential is well described by

$$
V(h)=\frac{1}{4} \lambda(h) h^{4},
$$

in the unitary gauge. The Higgs quartic coupling $\lambda(h)$ runs logarithmically with respect to $h$ from $\lambda\left(M_{h}\right) \simeq 0.13$ where $M_{h}$ is the Higgs mass. Here we consider the case that the Higgs potential has a maximum at $h=\Lambda_{0} \sim 10^{11} \mathrm{GeV}$ and becomes negative at slightly larger field values $h \gtrsim 10^{11}$ $\mathrm{GeV}$.

If the Hubble parameter during inflation ${ }^{1} H_{\text {inf }}$ is larger than $\Lambda_{0}$, the fluctuation of the Higgs field easily climbs up the potential barrier and rolls down to the unwanted true vacuum during inflation even when it initially sits at the origin [5]. The regions or the bubbles where the Higgs field falls into the unwanted true vacuum may collapse due to the AdS instability and hence only the regions where the Higgs field is inside the potential barrier may remain. Consequently the metastable electroweak vacuum and high-scale inflation may be compatible [5, 7]. However, it is not clear whether the Universe expands properly by inflation and the AdS bubble does not cause any cosmological disasters. In particular, if the AdS bubbles of the true vacuum "eat" the region where the present electroweak vacuum is selected, the existence of our Universe falls into a crisis [5, 7]. Therefore, we can say inflation with a relatively small Hubble parameter $H_{\text {inf }}<\Lambda_{0}$ is safe in the light of the current data of the Higgs and top mass.

The Higgs field can acquire a Hubble-induced mass due to its interaction with the inflaton $\phi$, for example, the "Higgs-portal" coupling $\kappa \phi^{2} h^{2} / 2$ or the non minimal coupling to gravity $\xi h^{2} R / 2$. Since $\phi \propto H$ in the case of chaotic inflation and $R \propto H^{2}$ during inflation and matter dominated era, these interactions can induce the "Hubble-induced" mass of the form

$$
\Delta V(h)=\frac{1}{2} c_{\text {inf }} H_{\mathrm{inf}}^{2} h^{2}
$$

with the coefficient $c_{\text {inf }}>0$ is determined by the coupling parameter such as $\kappa$ or $\xi$ in examples above. Here we consider the case $c_{\text {inf }} \lesssim \mathscr{O}(1)$ and study vacuum fluctuation in this potential. For $H_{\text {inf }} \gg \Lambda_{0}$, the Hubble-induced potential overwhelms the original potential around $h \sim \Lambda_{0}$ and the potential barrier moves to a higher field value. In principle, we should calculate the running of the couplings to study the dynamics of the Higgs field. However, they vary only logarithmically with respect to $h$ and hence we can treat them as constants, e.g., a negative quartic coupling $\lambda(h)=\tilde{\lambda} \simeq$ -0.01 , in the first approximation. Then, we obtain the field value at the potential barrier as

$$
\Lambda_{h} \simeq \sqrt{\frac{c_{\text {inf }}}{-\tilde{\lambda}}} H_{\mathrm{inf}}
$$

which is roughly ten times larger than the Hubble parameter during inflation for $c_{\text {inf }}=\mathscr{O}(1)$.

The Higgs field receives quantum fluctuations during inflation and acquires nonvanishing expectation value. If $c_{\text {inf }}$ is not too small, we can neglect the quartic term in the potential for the

\footnotetext{
${ }^{1}$ The subscript "inf" represents that the variable is evaluated at the inflationary era.
} 
Higgs field. If the Higgs field initially sits at the origin, the expectation value of the Higgs field is evaluated as [8]

$$
\left\langle h^{2}\right\rangle_{\text {inf }}=\frac{3 H_{\text {inf }}^{2}}{8 \pi^{2} c_{\text {inf }}}\left[1-\exp \left(-\frac{2 c_{\text {inf }}}{3} \mathscr{N}_{*}\right)\right],
$$

where $\mathscr{N}_{*}$ is the number of $e$-folds during inflation. It is still under discussion what is the correct survival condition [5, 7]: If the regions where experiences vacuum decay collapse into black holes and they evaporate quickly without destroying the stable electroweak vacua, vacuum decay during inflation is not dangerous (most optimistic case). On the other hand, if even only one region that experienced vacuum decay takes over all the space and dominates the Universe, a vacuum decay in the past light cone of the observable Universe causes catastrophe (most pessimistic case). Here we assume that the real survival condition lies between them and require $\left\langle h^{2}\right\rangle<\Lambda_{h}^{2}$ as its representative. Then, we acquire the constraint on the Hubble-induced mass as

$$
c_{\mathrm{inf}}>\sqrt{\frac{-3 \tilde{\lambda}}{8 \pi^{2}}} \simeq 1.9 \times 10^{-2}\left(\frac{\tilde{\lambda}}{-0.01}\right)^{1 / 2},
$$

regardless of the Hubble parameter during inflation. Here we have approximated $1-\exp \left(-2 c_{\text {inf }} \mathscr{N}_{*} / 3\right)$ $\simeq 1$. Note that Eq. (2.4) neglects the quartic term in the potential, and hence at the boundary values of $c_{\text {inf }}$ in Eq. (2.5), this approximation is no longer valid. The expectation value should be a little larger. However, the validity of this approximation recovers for a little larger value of $c_{\text {inf }}$. In this sense, Eq. (2.5) gives a most optimistic constraint that can be used as a reference.

Strictly speaking, we must calculate the evolution of the fluctuations with respect to the potential we have. For this purpose we have performed numerical calculation to solve the Langevin equations and found the approximation discussed in the above gives a good approximation. For example, Fig. 1 shows the histogram of the Higgs field value at $\mathscr{N}_{*}=50$ for $c_{\text {inf }}=10^{-2}, 10^{-1.5}, 10^{-1}$, $10^{-0.5}, 1$ and $\tilde{\lambda}=-0.01$, with $10^{5}$ trials. We find that for $c_{\text {inf }}>0.1$, the distribution is fitted by the Gaussian function with Eq. (2.4) $\left(\mathscr{N}_{*} \rightarrow \infty\right)$. We can find for the "just enough inflation", $\mathscr{N}_{*}=50$, the expectation value of the Higgs field is well described by Eq. (2.4) with $\mathscr{N}_{*}=\infty$. Therefore, we conclude that for the parameter that satisfies Eq. (2.5), the probability for the Higgs field to sit inside the potential barrier during inflation is not suppressed exponentially and it gives an appropriately optimistic condition for the survival of the electroweak vacuum. Hereafter we use Eq. (2.4) with $\mathscr{N}_{*}=\infty$ as a representative constraint.

\section{Dynamics of the Higgs field after inflation}

Now we turn to the Higgs field dynamics after inflation. Since the expectation value of the Higgs field just after inflation can be larger than the zero-temperature barrier $\Lambda_{0}$, we must consider the condition for the Higgs field to settle down to the electroweak vacuum through the dynamics after inflation.

Let us consider a case where the Higgs field still receives a positive Hubble-induced mass during inflaton oscillation dominated phase, ${ }^{2}$

$$
\Delta V(h)=\frac{1}{2} c_{\mathrm{osc}} H(t)^{2} h^{2},
$$

\footnotetext{
${ }^{2}$ The subscript "osc" represents that the parameter is evaluated at the inflaton oscillation dominated era.
} 


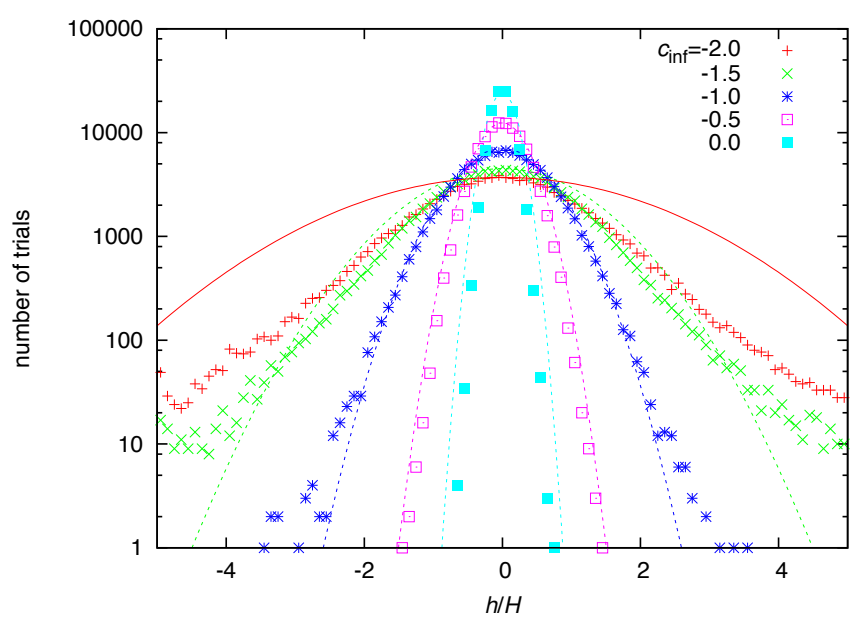

Figure 1: The histogram of the Higgs field value at $\mathscr{N}_{*}=50$ with $10^{5}$ trials. Dotted lines represent the Gaussian fitting, $\rho \propto \exp \left[-h^{2} / 2\left\langle h^{2}\right\rangle_{\text {inf }}\right]$, with $\mathscr{N}_{*} \rightarrow \infty$ (Eq. (2.4)). This figure is taken from Ref. [1].

where $c_{\text {osc }} \lesssim \mathscr{O}(1)$ is a numerical parameter. This is the case of the Higgs portal coupling with chaotic inflation as well as the non minimal coupling to gravity during the inflaton oscillation in the quadratic potential.

The Hubble parameter during inflaton oscillation dominated phase is well-approximated as $H(t)=2 / 3 t$. During this phase before the complete reheating, partial decay of inflaton produces relativistic particles as a subdominant component of the Universe. If their scattering cross section is large enough, they are thermalized with a temperature $T(t)=\left(72 / 5 \pi^{2} g_{*}(T)\right)^{1 / 8}\left(H(t) M_{\mathrm{Pl}} T_{R}^{2}\right)^{1 / 4}$, where $g_{*}$ is the effective number of relativistic degrees of freedom, and $T_{R}$ is the reheating temperature. We here assume that at least the fields that do not have direct couplings to the Higgs such as gluons are thermalized just after inflation.

The Higgs field is thermalized when the particles that couples to Higgs field becomes light enough, $h(t)<T(t)$, and the interaction rate is rapid enough, $\Gamma \sim T(t)>H(t)$. Thus, if these conditions are satisfied just after inflation, the Higgs field is thermalized quickly and the present Universe will be realized. If the reheating temperature is not high enough, the Higgs field is not thermalized just after the end of the inflation. Thus it takes some time for the Higgs field to be thermalized.

Let us study the dynamics of the Higgs field before thermalization. The Higgs field evolves according to the potential

$$
V(h)=\frac{1}{2} c_{\mathrm{osc}} H^{2}(t) h^{2}+\frac{1}{4} \lambda(h) h^{4}
$$

This potential has a time-varying maximum at $h=\Lambda_{t} \simeq \sqrt{\frac{c_{\text {osc }}^{-\lambda}}{-\lambda}} H(t)$, for $\Lambda_{t}>\Lambda_{0}$ where $\lambda \simeq$ $-\mathscr{O}\left(10^{-2}\right)$ is negative. Thus, for the healthy realization of the present Universe, $h(t)<\Lambda_{t}$ must be satisfied in the course of the evolution of the Higgs field in substantial part of the Universe. Otherwise the Higgs field rolls down towards the unwanted AdS vacuum in many regions of the Universe, which may cause a cosmological disaster. 
Now we evaluate the Higgs field dynamics taking $\left\langle h^{2}\right\rangle_{\text {inf }}^{1 / 2}$ (Eq. (2.4)) with $\mathscr{N}_{*} \rightarrow \infty$ as the initial condition. If the Higgs field does not roll down towards the unwanted AdS vacuum from this initial condition until its thermalization, the Higgs field successfully settles down to the electroweak vacuum in many regions of the Universe. Here, we require this as the survival condition.

The equation of motion (EOM) is given by

$$
\ddot{h}(t)+3 H(t) \dot{h}+c_{\mathrm{osc}} H^{2}(t) h(t)+\lambda(h) h^{3}(t)=0 .
$$

As long as $h(t)<\Lambda_{t}$ is satisfied, we can neglect the last term in the EOM and get a solution,

$$
h(t)=\left\langle h^{2}\right\rangle_{\mathrm{inf}}^{1 / 2}\left(\frac{H(t)}{H_{\mathrm{inf}}}\right)^{\left(1-\sqrt{1-16 c_{\mathrm{osc}} / 9}\right) / 2} \simeq \sqrt{\frac{3}{2 c_{\mathrm{inf}}}} \frac{H_{\mathrm{inf}}}{2 \pi}\left(\frac{H(t)}{H_{\mathrm{inf}}}\right)^{\left(1-\sqrt{1-16 c_{\mathrm{osc}} / 9} / 2\right.} .
$$

Here we consider the case where $c_{\text {osc }}<9 / 16$ and the Higgs field does not oscillate. Since the Higgs field value decreases slower than the potential barrier, $\Lambda_{t}$, we must seek for the way to avoid for the Higgs field to be caught up by the potential barrier after inflation for the successful Universe. Otherwise it rolls down to the unwanted AdS vacuum. This catching up would happen when

$$
h(t) \simeq \Lambda_{t} \Leftrightarrow H(t) \simeq\left(\frac{-3 \lambda}{8 \pi^{2} c_{\mathrm{osc}} c_{\mathrm{inf}}}\right)^{1 /\left(1+\sqrt{1-16 c_{\mathrm{osc}} / 9}\right)} H_{\mathrm{inf}} \equiv H_{c} .
$$

The first way to avoid the falling down to the unwanted AdS vacuum is that the Higgs field gets thermalized before being caught up. We can take the criteria for the Higgs field thermalization as $T(t)>h(t)$ and $T(t)>H(t)$. After some calculations, we will have the lower bound of the reheating temperature for the successful Universe for a given Hubble parameter during inflation and the coefficients $c_{\text {inf/osc }}$.

The second way for the successful cosmic history is that the Higgs field value $h(t)$ becomes smaller than the zero-temperature barrier $\Lambda_{0}$ and gradually its dynamics is dominated by $\lambda h^{4} / 4$ term before being caught up by the potential barrier. The Higgs expectation value $h(t)$ gets smaller than $\Lambda_{0}$ when

$$
H(t)<\left(\sqrt{\frac{8 \pi^{2} c_{\text {inf }}}{3}} \frac{\Lambda_{0}}{H_{\text {inf }}}\right)^{2 /\left(1-\sqrt{1-16 c_{\text {osc }} / 9}\right)} H_{\text {inf }} \equiv H_{\Lambda} .
$$

Thus, if $H_{\Lambda}>H_{c}$, the present electroweak vacuum is successfully selected. This condition gives a constraint on the Hubble parameter during inflation as

$$
H_{\text {inf }}<\left(\frac{8 \pi^{2} c_{\text {inf }}}{3}\right)^{1 / 2}\left(\frac{-3 \lambda}{8 \pi^{2} c_{\text {inf }} c_{\text {osc }}}\right)^{\frac{1+\sqrt{1-16 c_{\text {osc }} / 9}}{1-\sqrt{1-16 \operatorname{cosc} / 9}}} \Lambda_{0}
$$

By taking into account these two cases without cosmological disaster, one can determine the cosmic histories, parameterized by the Hubble induced mass $c_{\text {inf/osc }}$, the Hubble parameter during inflation $H_{\text {inf }}$ and the reheating temperature $T_{R}$. As an example, we show the allowed region in the $T_{R^{-}} c_{\text {inf }}$ plane in Figs. 2 for $H_{\text {inf }}=10^{12}, 10^{13}$ and $10^{14} \mathrm{GeV}$, respectively, with the parameters being chosen as $\Lambda_{0}=10^{11} \mathrm{GeV}$, and $c_{\text {osc }}=c_{\text {inf }} / 2$ (Fig. 2). The thick red colored region is disfavored due to the condition $\left\langle h^{2}\right\rangle_{\text {inf }}>\Lambda_{h}^{2}$ with Eq. (2.4) $\left(\mathscr{N}_{*} \rightarrow \infty\right)$, which means the survival probability 


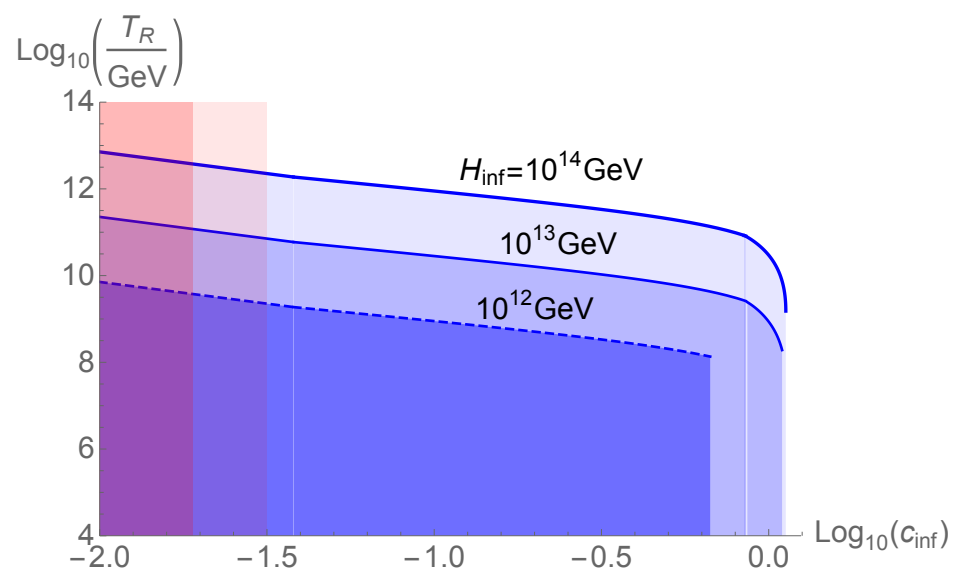

Figure 2: The constraint on the reheating temperature according to the coupling constant $c_{\text {inf }}$ with $c_{\text {osc }}=$ $c_{\text {inf }} / 2$. This figure is taken from Ref. [1].

during inflation is exponentially suppressed. We also show the constraint $c_{\text {inf }}<10^{-1.5}$ that represents $\left\langle h^{2}\right\rangle_{\text {inf }}<\Lambda_{h}^{2}$ for $\mathscr{N}_{*} \simeq 100$ in light red region. The blue colored regions are excluded due to the condition for the Higgs field not to fall into the unwanted true vacuum. Note that there are no constraint for $c_{\text {inf }}>10^{-0.17}\left(10^{0.04}\right)\left(c_{\text {osc }}=c_{\text {inf }} / 2\right)$ in the cases $H_{\text {inf }}=10^{12(13)} \mathrm{GeV}$, in which the condition $h(t)<\Lambda_{0}$ is always satisfied before the rolling down to the unwanted true vacuum. We can see that the lower bound of the reheating temperature becomes severer as the Hubble parameter during inflation is larger. For the Hubble parameter $H_{\text {inf }} \simeq 10^{14} \mathrm{GeV}$, a relatively high reheating temperature, $T_{R} \gtrsim 10^{12-13} \mathrm{GeV}$ is required. This indicates that if the $\mathrm{B}$-mode in the CMB polarization observation with $r \simeq 0.1$ is confirmed, the stochastic GW background must be detected in the gravitational detectors [9] such as DECIGO or BBO due to the relatively large reheating temperature. If not, it suggests that there is a physics beyond the SM to stabilize the Higgs potential or the Hubble-induced mass for the Higgs field during inflation is much larger than the Hubble parameter.

\section{Summary}

In this article, we studied the evolution of the SM Higgs field in the inflationary cosmology in the light of recent collider experiments, which suggests the metastability of the electroweak vacuum. If the electroweak vacuum is metastable, high-scale inflation may be problematic since the Higgs field rolls down to the unwanted AdS vacuum and the probability for the Higgs field to remain the electroweak vacuum is exponentially suppressed, though it is still under discussion if it is a real catastrophe for our Universe or not $[5,7]$. We found that the Hubble-induced mass can avoid the exponentially suppressed survival probability of the electroweak vacuum during inflation while it is not necessarily larger than the Hubble parameter during inflation if the number of $e$ folds during inflation is not too large. We also found that the present Universe can be successfully realized even in the case of the relatively small Hubble-induced mass if the reheating temperature is high enough. This is because the Higgs field is thermalized before being caught up by the timedependent potential barrier and before rolling down to the unwanted AdS vacuum. As a result, relatively high-energy scale inflation is allowed, and hence we can expect for the detection of GW 
background in the future experiments. We also pointed out that the direct GW background detection will give us the clue to study the physics beyond the SM. Note that since the Higgs mass during inflation can be smaller than the Hubble parameter, it may be possible to generate a feature in the $\mathrm{CMB}$, for example, nongaussianity, though it will require nontrivial interaction for the Higgs field.

\section{Acknowledgments}

The author is grateful to M. Asano, O. Lebedev, and A. Westphal for collaboration at the early stage of this project. The author also thanks M. Shaposhnikov for useful comments. This work has been supported in part by the JSPS Postdoctoral Fellowships for Research Abroad. The author also acknowledges support from the DOE for this work under Grant No. DE-SC0013605.

\section{References}

[1] K. Kamada, Inflationary cosmology and the standard model Higgs with a small Hubble induced mass, Phys. Lett. B 742, 126 (2015) [arXiv:1409.5078 [hep-ph]].

[2] G. Aad et al. [ATLAS Collaboration], Observation of a new particle in the search for the Standard Model Higgs boson with the ATLAS detector at the LHC,' Phys. Lett. B 716, 1 (2012) [arXiv:1207.7214 [hep-ex]].

[3] S. Chatrchyan et al. [CMS Collaboration], Observation of a new boson at a mass of $125 \mathrm{GeV}$ with the CMS experiment at the LHC, Phys. Lett. B 716, 30 (2012) [arXiv:1207.7235 [hep-ex]].

[4] [Tevatron Electroweak Working Group and CDF and D0 Collaborations], Combination of CDF and $D 0$ results on the mass of the top quark using up to $5.8 \mathrm{fb}-1$ of data, arXiv:1107.5255 [hep-ex]; [ATLAS and CDF and CMS and D0 Collaborations], First combination of Tevatron and LHC measurements of the top-quark mass, arXiv:1403.4427 [hep-ex].

[5] J. R. Espinosa, G. F. Giudice and A. Riotto, Cosmological implications of the Higgs mass measurement, JCAP 0805, 002 (2008) [arXiv:0710.2484 [hep-ph]].

[6] P. B. Arnold, Can The Electroweak Vacuum Be Unstable?, Phys. Rev. D 40, 613 (1989); M. Sher, Electroweak Higgs Potentials and Vacuum Stability, Phys. Rept. 179, 273 (1989).

[7] A. Hook, J. Kearney, B. Shakya and K. M. Zurek, Probable or Improbable Universe? Correlating Electroweak Vacuum Instability with the Scale of Inflation, JHEP 1501 (2015) 061 [arXiv:1404.5953 [hep-ph]].

[8] T. S. Bunch and P. C. W. Davies, Quantum Field Theory in de Sitter Space: Renormalization by Point Splitting, Proc. Roy. Soc. Lond. A 360, 117 (1978).

[9] K. Nakayama, S. Saito, Y. Suwa and J. Yokoyama, Space laser interferometers can determine the thermal history of the early Universe, Phys. Rev. D 77, 124001 (2008) [arXiv:0802.2452 [hep-ph]]; K. Nakayama, S. Saito, Y. Suwa and J. Yokoyama, Probing reheating temperature of the universe with gravitational wave background, JCAP 0806, 020 (2008) [arXiv:0804.1827 [astro-ph]]. 